\title{
DISCRIMINAÇÃO ENTRE LOTES DE SEMENTES DE GIRASSOL ATRAVÉS DO TESTE DE ENVELHECIMENTO RÁPIDO (1)
}

\author{
JOCELY ANDREUCCETTI MAEDA $(2,4)$, LUIZ FERNANDES RAZERA $(2,4)$, \\ ANTONIO AUGUSTO DO LAGO $(2,4)$ \\ E MARIA REGINA GONÇALVES UNGARO $(3,4)$
}

\begin{abstract}
RESUMO
Vinte lotes distintos de sementes de girassol foram submetidos ao envelhecimento rápido a $42{ }^{\circ} \mathrm{C}$ e $100 \%$ de umidade relativa, por 0,24 , $48,72,96$ e 120 horas, com o objetivo de determinar o tempo de exposição que melhor discriminasse os lotes pelas suas repostas de germinação após o envelhecimento. Os resultados mostraram que os lotes exibiram grandes diferenças entre si quanto ao grau de resistência às condições desfavoráveis impostas pelo envelhecimento rápido, inclusive aqueles com porcentagens comparáveis de germinação inicial. Esse comportamento variável não pôde ser estimado pelo teste inicial de germinação. O periodo de 72 horas de exposição na câmara foi o que ofereceu melhor discriminação entre os lotes de germinação inicial mais alta. Entre os lotes de menor poder germinativo inicial (menos de 50\%), o periodo que proporcionou melhor discriminação foi o de 48 horas de exposição.
\end{abstract}

Termos de indexação: germinaçāo, vigor, girassol, Helianthus annus $\mathrm{L}$.

(1) Recebido para publicação em 26 de junho de 1985.

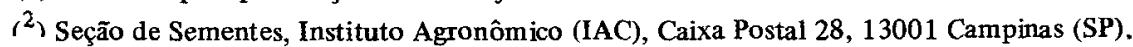

(3) Seção de Oleaginosas, IAC.

( ${ }^{4}$ ) Com bolsa de suplementa ção do $\mathrm{CNPq}$. 


\section{INTRODUÇÃO}

Sementes de alta qualidade fisiológica ou de alto índice de vigor apresentam maior longevidade durante o período de armazenamento e, após semeadas, germinam e emergem rápida e uniformemente, produzindo plantas sadias, bem desenvolvidas e de maior produtividade. Devido a diferenças de vigor ainda não adequadamente avaliadas, lotes de sementes da mesma espécie, variedade, idade cronológica e porcentagem de germinação podem exibir comportamento variável, mesmo quando armazenados ou semeados nas mesmas condições (HELMER, 1962).

A qualidade fisiológica das sementes é ainda universalmente avaliada pelo teste padrão de germinação, cuja validade tem sido grandemente questionada e, ạtualmente, pesquisadores, agricultores e produtores de sementes mostram-se muito interessados no desenvolvimento de um teste mais adequado para avaliar o vigor de lotes de sementes.

GRABE (1965), BYRD \& DELOUCHE (1971) e DELOUCHE \& BASKIN (1973) explicaram essa deficiência do teste padrão, afirmando que a perda de germinação é um dos últimos efeitos do processo de deterioração, podendo, então, um lote de sementes sofrer um substancial decréscimo em vigor, sem exibir um correspondente decréscimo em porcentagem de germinação.

Entre os diversos testes desenvolvidos e estudados para determinar o vigor de sementes, o do envelhecimento rápido parece ser um dos mais promissores, pois, além da sua efetividade na avaliação do vigor (potencial de armazenamento e comportamento no campo), é também de execução relativamente simples, o que facilita a sua introdução nas análises de rotına pelos laboratórios de sementes.

Esse teste consiste basicamente em submeter as sementes a altas temperaturas $(40-450 \mathrm{C})$ e alta umidade relativa $(100 \%)$ por determinado período seguido pelo teste padrão de germinação (POPINIGIS, 1977).

DELOUCHE \& BASKIN (1973) afirmaram que os processos de deterioração sob condições de envelhecimento rápido são semelhantes àqueles que ocorrem sob condições normais, com a diferença de que a velocidade de deterioração é grandemente aumentada. Os mesmos autores, baseados em investigações envolvendo lotes de inúmeras espécies de sementes, mostraram que as porcentagens de germinação após o envelhecimento rápido estavam altamente associadas com a armazenabilidade e com o comportamento após plantio no campo.

Entre as inúmeras espécies de sementes ainda carentes de melhores estudos com relação ao teste de envelhecimento rápido, estão as de girassol, cuja cultura encontra no Brasil uma área favorável extremamente ampla, com 
grande potencial de desenvolvimento e de vantagens econômicas para agricultores e industriais. $E$ interessante ressaltar que essa semente contém cerca de $45 \%$ de óleo comestível (UNGARO, 1985) de alto valor dietético e ao redor de $20 \%$ de proteínas de alto valor biológico (DORREL, 1978).

MAEDA et alii (1985) submeteram seis diferentes lotes de sementes de girassol ao teste de envelhecimento rápido a $42{ }^{\circ} \mathrm{C}$ por períodos que variaram de 0 a 120 horas: concluíram que o período de 72 horas foi o que melhor discriminou níveis de vigor entre lotes, e sugeriram que um número ainda maior de lotes deveria ser estudado para confirmar de maneira mais segura aquelas conclusões.

Seguindo a sugestão de MAEDA et alii (1985), o presente estudo foi levado a efeito com o objetivo de encontrar a melhor forma de discriminação entre lotes de sementes dessa oleaginosa quanto às respostas de germinação após diversos períodos de envelhecimento rápido.

\section{MATERIAL E MÉTODOS}

O experimento foi realizado utilizando-se vinte lotes de sementes de girassol provenientes do Banco de Germoplasma da Seção de Oleaginosas do Instituto Agronômico. A relação dos lotes, cultivares e respectivas procedências consta do quadro 1.

No teste de envelhecimento rápido, amostras representativas dos vinte lotes foram mantidas em câmara especial para o teste, com temperatura constante de $42^{\circ} \mathrm{C}$ e umidade relativa de $100 \%$ por $0,24,48,96$ e 120 horas (HELMER, 1962).

Após cada período, uma amostra de cada um dos vinte lotes foi retirada da câmara e submetida ao teste padrão de germinação (BRASIL, 1980), utilizando-se oito repetições de cinqüenta sementes cada uma.

Durante a tomada dos dados, notou-se que seis dos lotes apresentavam, a zero hora de exposição, germinação abaixo de $50 \%$. Tais lotes foram analisados em separado dos outros 14, pois sua exagerada queda de germinação e a maior variabilidade entre repetições, normal em sementes de baixa germinação e vigor, poderiam distorcer os resultados com relação aos outros lotes de maior poder germinativo e vigor. Além disso, em estudos de vigor de sementes, é interessante que os lotes apresentem porcentagens iniciais de germinação não muito diferentes entre si.

A análise estatística dos dados de germinação após os diversos períodos de envelhecimento foi feita com a finalidade de verificar o período que melhor discriminou os lotes, ou seja, o periodo que os classificou em maior número de grupos de lotes de qualidade fisiológica (vigor) diferente. 
Quando da comparação de médias (GOMES, 1966), cada grupo foi formado por lotes unidos pela mesma letra e, portanto, com níveis de vigor estatisticamente iguais.

QUADRO 1. Cultivares utilizados e respectivas procedências, no Estado de São Paulo

\begin{tabular}{|c|c|c|}
\hline $\begin{array}{c}\text { Lote } \\
\text { no }\end{array}$ & Cultivar & Procedência $\left({ }^{1}\right)$ \\
\hline 1 & IAC-Anhandy & Campinas \\
\hline 2 & IAC-Anhandy & Campinas \\
\hline 3 & IAC-Anhandy & Tatuí \\
\hline 4 & IAC-Anhandy & Campinas \\
\hline 5 & IAC-Anhandy & Campinas \\
\hline 6 & IAC-Anhandy & Campinas \\
\hline 7 & IAC-Anhandy & Mococa \\
\hline 8 & Contisol-812 & Ribeirão Preto \\
\hline 9 & DK-180 & Barretos \\
\hline 10 & Uruguai & Jaủ \\
\hline 11 & VNIIMK & Mococa \\
\hline 12 & Uruguai & São Roque \\
\hline 13 & IAC-Anhandy & Campinas \\
\hline 14 & IAC-Anhandy & Campinas \\
\hline 15 & Apollo & Monte Alegre do Sul \\
\hline 16 & GH-7811 & Ribeirão Preto \\
\hline 17 & HS-90 & Monte Alegre do Sul \\
\hline 18 & Airelli & Ribeirão Preto \\
\hline 19 & Uruguai & Jundiaí \\
\hline 20 & Florestal Cambá & Pindorama \\
\hline
\end{tabular}

( ${ }^{1}$ ) Com exceção das sementes provenientes da região de Barretos, todas as demais foram obtidas em campos de produção de sementes de Estaçōes Experimentais do Instituto Agronômico de Campinas. 


\section{RESULTADOS E DISCUSSÃO}

Os resultados, com relação aos lotes de maior germinação inicial, encontram-se no quadro 2. Como esperado, o poder germinativo decresceu com o aumento do número de horas de exposição na câmara de envelhecimento. Em alguns lotes, esse descréscimo foi maior que em outros. Como exemplo, enquanto o lote nọ 7 decresceu apenas com 20,0\% em germinação após 120 horas, o nọ 8 decresceu $71,0 \%$ depois do mesmo período.

QUADRO 2. Resultados do teste de envelhecimento rápido $\left(42^{\circ} \mathrm{C}\right.$ e cerca de $100 \%$ de umidade relativa) em lot es de sementes de girassol

\begin{tabular}{|c|c|c|c|c|c|c|}
\hline \multirow{2}{*}{$\begin{array}{c}\text { Lote } \\
\text { no }\end{array}$} & \multicolumn{6}{|c|}{ Porcentagens de germinação após os períodos de tratamento (horas) } \\
\hline & 0 & 24 & 48 & 72 & 96 & 120 \\
\hline 1 & $\left.84,1 \operatorname{cde}^{2}\right)$ & 76,6 de & $48,0 \mathrm{e}$ & $46,5 \mathrm{~g}$ & $33,5 \mathrm{e}$ & $21,4 \mathrm{~d}$ \\
\hline 2 & 85,4 bcd & 76,1 de & $59,0 \mathrm{e}$ & $52,3 \mathrm{fg}$ & $28,0 \mathrm{e}$ & 17,7 de \\
\hline 3 & 78,1 de & 68,3 ef & $56,0 \mathrm{e}$ & $51,0 \mathrm{fg}$ & $25,3 \mathrm{e}$ & $26,7 \mathrm{~d}$ \\
\hline 4 & $89,6 \mathrm{abc}$ & $89,5 \mathrm{abc}$ & 78,5 bed & $72,0 \mathrm{~cd}$ & 73,5 bc & $54,8 b c$ \\
\hline 5 & 92,3 ab & 93,3 a & $84,6 \mathrm{abc}$ & 88,8 a & 86,3 a & 76,1 a \\
\hline 6 & $92,4 \mathrm{ab}$ & $91,1 \mathrm{ab}$ & $87,2 \mathrm{ab}$ & 88,1 a & 85,5 a & $71.8 \mathrm{a}$ \\
\hline 7 & 92,6 a & $91,0 \mathrm{ab}$ & 87,4 a & 89,5 a & 84,6 a & $72,6 \mathrm{a}$ \\
\hline 8 & $79,2 \mathrm{de}$ & $54,8 \mathrm{~g}$ & 36,5 \& & $29,5 \mathrm{~h}$ & $9,4 \mathrm{f}$ & 8,2 e \\
\hline 9 & $89,9 \mathrm{abc}$ & $85,6 \mathrm{bc}$ & $86,5 \mathrm{abc}$ & $81,8 \mathrm{ab}$ & $75,3 \mathrm{~b}$ & 72,1 a \\
\hline 10 & $92,5 \mathrm{a}$ & $82,6 \mathrm{~cd}$ & $77,8 \mathrm{~cd}$ & $76,4 \mathrm{bc}$ & $69,8 \mathrm{bc}$ & $67,9 \mathrm{ab}$ \\
\hline 11 & 88,8 abc & $83,3 \mathrm{~cd}$ & $70,0 \mathrm{~d}$ & $65,5 \mathrm{de}$ & $68,3 b c$ & $45,2 c$ \\
\hline 12 & $76,3 \mathrm{e}$ & $61,8 \mathrm{fg}$ & $74,1 \mathrm{~d}$ & $25,5 \mathrm{~h}$ & $25,8 \mathrm{e}$ & $18,3 \mathrm{de}$ \\
\hline 13 & 80,6 de & 84,5 bcd & $78,0 \mathrm{~cd}$ & $73,0 \mathrm{bcd}$ & $63,9 \mathrm{c}$ & $52,0 \mathrm{c}$ \\
\hline 14 & 82,8 cde & 84,5 bcd & $80,4 \mathrm{abc}$ & 60,3 ef & $51,2 \mathrm{~d}$ & $42,7 \mathrm{c}$ \\
\hline$F\left({ }^{1}\right)$ & $14,15^{* *}$ & $43,20 * *$ & $58,02 * *$ & $81,27 * *$ & $172,26^{* *}$ & $70,98 * *$ \\
\hline $\begin{array}{l}\text { d.m.s. } \\
\text { (Tukey 5\%) }\end{array}$ & 6,55 & 6,20 & 6,66 & 6,63 & 6,33 & 8,95 \\
\hline C.V. (\%) & 3,78 & 3,82 & 4,51 & 15,29 & 5,17 & 8,38 \\
\hline
\end{tabular}

(1) Os valores de F, d.m.s. e C.V., assim como a comparaçăo de méd ias, săo correspondentes aos dados transformados em arco seno $\sqrt{\% / 100}$.

$\left(^{2}\right)$ Letras nâo comuns na coluna indicam diferenças significativas detectadas pelo teste de Tukey ao nível de $5 \%$.

A zero hora, a diferença entre a maior e a menor germinação entre os lotes foi apenas de $16,3 \%$. No entarito, à medida que o tempo de exposição aumentou, essa diferença também se elevou, atingindo o máximo de 76,9\% 
decorridas 96 horas. 0 aumento dessa diferença indicou grande variação entre os lotes quanto a sua resistência às condições desfavoráveis de temperatura e umidade relativa do teste; uma estimativa confiável dessa variação não seria possível tendo como base apenas os resultados do teste de germinação a zero hora (teste padrão de germinação).

Lotes de germinação inicial (a zero hora) semelhantes responderam diferentemente ao envelhecimento acelerado. Como exemplo, os lotes 4 e 9 , com germinação inicial de $89,6 \%$ e $89,9 \%$, respectivamente, apresentaram, após 120 horas, germinação de $54,8 \%$ e $72,1 \%$, respectivamente, revelando diferenças de comportamento entre os lotes, não detectadas pelo teste de germinação inicial.

A a nálise estatística dos dados (Quadro 2) demonstrou que, enquanto o período de zero hora classificou os 14 lotes em cinco grupos significativamente diferentes, os períodos de $24,48,72,96$ e 120 horas classificaram os mesmos lotes em 7, 6, 8, 6 e 5 grupos distintos respectivamente. Portanto, o período de 72 horas foi o que possibilitou a maior discriminação entre os lotes quanto a níveis de vigor. Assim, por exemplo, enquanto a germinação inicial dos lotes $4,5,6,7,9,10$ e 11 foi estatisticamente igual (formando um só grupo), após 72 horas de exposição, a germinação dos lotes, 5, 6, 7 e 9 foi estatisticamente superior à dos lotes 4 e 10, que, por sua vez, foi superior à do lote 11 .

O teste de envelhecimento por 120 horas foi o menos efic iente na discriminação entre os lotes.

No quadro 3 estão expostos os dados relacionados com o grupo de lotes de menor germinação inicial. A queda drástica de germinação desses lotes, verificada após 24 horas de exposição, não permite maiores considerações práticas quanto ao seu comportamento em relação ao envelhecimento acelerado. No entanto, o período de $\mathbf{4 8}$ horas parece ter sido o que melhor discriminou entre os seis lotes, classificando-os estatisticamente em quatro níveis, enquanto os períodos de $0,24,72,96$ e 120 horas classificaram os mesmos lotes em $3,3,3,1$ e 2 níve is respectivamente.

Dessa maneira, diante dos resultados obtidos, sugere-se o teste de envelhecimento acelerado a $42{ }^{\circ} \mathrm{C}$ por 72 horas para a avaliação do vigor de sementes de girassol com índice de germinação alto ou relativamente alto, e o mesmo teste a $42^{\circ} \mathrm{C}$ por 48 horas para os lotes de germinação baixa ou muito baixa (menos que $50 \%$ ).

ADAMO et alii (1984), estudando a curva de deterioração de sementes de girassol submetidas às condições de envelhecimento acelerado a $42^{\circ} \mathrm{C}$, observaram que .0 decréscimo em germinação foi mais acentuado após 48 horas de envelhecimento. Afirmaram eles ser esse o periodo mais apropriado para avaliar o vigor de sementes de girassol, embora tivessem utilizado apenas um lote em seus estudos. 
As observações de MAEDA et alii (1985) sobre o período de 72 horas a $42^{\circ} \mathrm{C}$ foram confirmadas no presente experimento, com relação a lotes de germinação alta ou relativamente alta.

QUADRO 3. Resultados do teste de envelhecimento rápido $\left(42^{\circ} \mathrm{C}\right.$ e cerca de $100 \%$ de umidade relativa) em lotes de sementes de girassol de baixo valor germinativo

\begin{tabular}{|c|c|c|c|c|c|c|}
\hline \multirow{2}{*}{$\begin{array}{c}\text { Lote } \\
\text { no }\end{array}$} & \multicolumn{6}{|c|}{ Porcentagem de germinação após os periodos de tratamento (hora) } \\
\hline & 0 & 24 & 48 & 72 & 96 & 120 \\
\hline 15 & $35,5 \mathrm{~b}\left({ }^{2}\right)$ & $3,1 \mathrm{c}$ & $1,0 \mathrm{~d}$ & $0,0 \mathrm{c}$ & $0,0 \mathrm{a}$ & $0,0 \mathrm{~b}$ \\
\hline 16 & $28,7 \mathrm{~b}$ & $13,1 \mathrm{ab}$ & $5,6 \mathrm{bc}$ & $3,4 \mathrm{ab}$ & 0,8 a & 1,2 a \\
\hline 17 & $49,7 \mathrm{a}$ & $21,7 \mathrm{a}$ & $16,3 \mathrm{a}$ & $5,0 \mathrm{a}$ & $1,6 \mathrm{a}$ & $1,3 \mathrm{a}$ \\
\hline 18 & 45,5 a & 23,8 a & $20,3 \mathrm{a}$ & 7,4 a & $2,2 \mathrm{a}$ & $0,6 \mathrm{ab}$ \\
\hline 19 & $32,5 \mathrm{~b}$ & $10,2 \mathrm{~b}$ & $12,0 \mathrm{ab}$ & $0,9 \mathrm{bc}$ & $2,0 \mathrm{a}$ & $0,0 \mathrm{~b}$ \\
\hline 20 & $16,9 \mathrm{c}$ & $6,8 \mathrm{bc}$ & $4,6 \mathrm{~cd}$ & $0,8 \mathrm{bc}$ & $1,0 \mathrm{a}$ & $0,0 \mathrm{~b}$ \\
\hline$F\left({ }^{1}\right)$ & $44,73 * *$ & $16,46 * *$ & $20,89 * *$ & $14,35 * *$ & $1,66^{\text {n.s. }}$ & $5,65 * *$ \\
\hline $\begin{array}{l}\text { d.m.s. } \\
\text { (Tukey 5\%) }\end{array}$ & 4,94 & 8,15 & 7,79 & 6,04 & 7,50 & 3,37 \\
\hline C.V. (\%) & 6,17 & 17,86 & 20,32 & 30,74 & 53,35 & 34,72 \\
\hline
\end{tabular}

( ${ }^{1}$ ) Os valores de F, d.m.s. e C.V., assim como a comparaçâo de médias, são correspondentes aos dados transformados em arco seno $\sqrt{\% / 100}$.

$\left({ }^{2}\right)$ Letras não comuns na coluna indicam diferenças significativas detectadas pelo teste de Tukey ao nível de $5 \%$.

\section{CONCLUSÕES}

a) Os lotes de sementes de girassol exibiram grandes diferenças entre si quanto ao grau de resistência às condições desfavoráveis impostas pelo envelhecimento rápido. Esse comportamento variável não pôde ser estimado pelo teste inicial de germinação. 
b) 0 período de 72 horas de exposição ao envelhecimento rápido foi o que melhor discriminou os lotes que apresentavam maior germinação inicial.

c) Nos lotes de menor poder germinativo inicial, o período que proporcionou melhor discriminação foi o de 48 horas de exposição.

\title{
SUMMARY
}

\section{DISCRIMINATION AMONG SUNFLOWER SEED LOTS BY THE ACCELERATED AGING TEST}

\begin{abstract}
Twenty sunflower seed lots were subjected to accelerated aging at $42 \circ \mathrm{C}$ and $100 \%$ relative humidity for periods of $0,24,48,72,96$, and 120 hours. The objective was to determine the exposure time which best discriminated the lots as to the germination response after aging. Results showed that seed lots were quite different concerning the degree of resistance to the unfavorable conditions of accelerated aging, even those with comparable initial percentages of germination. The initial standard germination test was not appropriate to show differences among lots. The 72 hour exposure period was the best for discriminating lots with higher percentages of germination, and the 48 hours exposure period was the best for lots with lower percentages of germination (less than $50 \%$ ).
\end{abstract}

Index terms: germination, vigor, sunflower, Helianthus annuus L.

\section{REFERÊNCIAS BIBLIOGRÁFICAS}

ADAMO, P.E.; SADER, R. \& UNGARO, M.R.G. Comportamento germinativo de sementes de girassol submetidas ao teste de envelhecimento precoce. Revista Brasileira de Sementes, Brasflia, 6(3):15-20, 1984.

BRASIL. Ministério da Agricultura. Divisão de Sementes e Mudas, Regras para anátise de sementes. Brastlia, 1980. 188p.

BYRD, H.W. \& DELOUCHE, J.C. Deterioration of soybean seed in storage. Proceedings of the Association of Official Seed Analysts, 61:41-57, 1971.

DELOUCHE, J.C. \& BASKIN, C.C. Accelerated aging techniques for predicting the relative storability of seed lots. Seed Science and Technology, 1:427-452, 1973.

DORREL, D.G. Processing and utilization of oilseed sunflower. In: CARTER, J.F, ed. Sunflower science and technology. Madison, American Society of Agronomy, 1978. p.407-440. (Agronomy, 19) 
GOMES, F.P. Curso de estatistica experimental. 3.ed. Piracicaba, ESALQ, 1966. 404p.

GRABE, D.F. Prediction of relative storability of corn seed lots. Proceedings of the Association of Official Seed Analysts, 55:92-96, 1965.

HELMER, J.D. Evaluation of some methods for differentiating among vigor levels in seeds of crimson and red clover. Mississippi State, M.S.U., 1962. 62p. Tese (Mestrado)

MAEDA, J.A.; RAZERA, L.F. \& UNGARO, M.R.G. Sementes de girassol: observaçöes preliminares sobre 0 teste de envelhecimento rápido. Bragantia, Campinas, 44(1):417-420, 1985.

POPINIGIS, F. Fisiologia da semente. Brastlia, Ministério da Agricultura, AGIPLAN, 1977. 289p.

UNGARO, M.R.G. Instruçōes para a cultura do girassol. Campinas, Instituto Agronômico, 1985. 18p. (Boletim técnico, 103) 